\title{
Toroidicity effects on the absorption of IC waves by the electrons
}

\author{
M. Brambilla, R. Bilato \\ Max Planck Institute for Plasma Physics, Boltzmannstr. 2, 85748 Garching, \\ Germany
}

\begin{abstract}
The poloidal modulation of the parallel velocity of charged particles in the tokamak magnetic field modifies the strength of electron absorption of the compressional wave in the Ion Cyclotron (IC) range of frequencies. We have developed a model of this effect which is sufficiently simple to be numerically tractable, and we have implemented it in the TORIC full-wave code, which solves the wave equation in toroidal axisymmetric geometry. We find that the effect is normally negligible in present-day devices, but its importance increases with the dimensions and the temperature of the plasma, and should be taken into account when estimating the competition between electron and ion heating and the efficiency of current drive in future reactor-grade plasmas.
\end{abstract}

\section{Introduction}

That toroidicity influences absorption of the compressional wave (also known as magnetosonic, or fast wave $(\mathrm{FW})$ ) by the electrons in the Ion Cyclotron (IC) and magnetohydrodynamic (MHD) range of frequencies is known since some time $[1,2,3,4,5,6,7]$. These authors have pointed out that the poloidal modulation of the parallel velocity of charged particles modifies the longitudinal permeability of the plasma (whose imaginary part describes electron Landau damping of electrostatic waves). Grishanov and co-workers [8,9] have specialized these calculations to two particular tokamak configurations. To the best of our knowledge, however, these effects have never yet been taken into account in a full wave solver in toroidal geometry. The extension of the theory to all the electron contributions to the dielectric tensor, so that it can be applied also to a wave which is not purely electrostatic, is straightforward, but the 'exact' expressions for the coefficients of the wave equations modified following the authors cited above are so cumbersome that it is next to impossible to code them efficiently. To quantify the influence of toroidicity on electron damping in realistic configurations, we have now implemented an approximate formulation of these effects in the code TORIC [10]. 
A first effect of toroidicity, namely the broadening of the Cherenkov-Landau resonance due to the combination of the normal Doppler effect with the finite length of the resonance of the individual electrons, was discussed in [11]. For the estimate of the efficiency of direct electron heating and current drive, however, it is even more important to take into account the effects of the modulation of the electrons parallel velocity by the inhomogeneous static magnetic field on the location and duration of these resonances. This is the object of the present study.

As a preliminary, in the next section we summarize the contributions of the electrons to the high-frequency (hf) current, i.e. to the coefficients of the wave equations. As just stated, our goal is to take into account the toroidal modulation of the electron parallel velocity in the evaluation of these coefficients. For this purpose, in section 3 we briefly recall the features of the motion of charged particles in the tokamak magnetic field relevant for our problem. We then introduce the approximations we have found necessary in order to make the problem numerically tractable. In summary, they consist in neglecting the contribution of trapped electrons altogether, and in approximating the effects of toroidicity on the motion of passing particles as a sinusoidal perturbation, which can then be treated in much the same way as the gyration motion when evaluating the orbit integrals in the solution of the linearized Vlasov equation. We will argue that these approximations are much less drastic than it might appear from their formulation here. In the final section we present a few examples of results from our model implemented in TORIC.

In medium size devices with electron temperature not exceeding a few $\mathrm{keV}$ absorption of the compressional, or fast wave ( $\mathrm{FW})$, by electrons is usually very weak compared to absorption by ions at IC resonances; its modification by toroidal effects is, therefore, almost irrelevant. The importance of these modifications, however, can be expected to increase with the temperature and the dimensions of the plasma. Indeed, in large and hot fusion plasmas electron damping will be a more serious competitor; and the fact that the FW will have to travel through a large plasma volume before reaching IC resonances will enhance the effects of moderate changes in the local electron damping, thereby allowing toroidicity effect to appreciably alter the global balance between electron and ion heating.

The direction of these modifications depends on the parallel phase velocity of the waves (cf. the comments after eqns (31) in section 3), and thus on the toroidal spectrum launched by the antenna. For partial waves in the main peak of the typical spectrum tailored for IC heating or current drive the main local change is due to the fact that the duration of the Cerenkov resonance of individual electrons is maximum when they occur near the outer equatorial plane, i.e. just below the antenna. This tends to increase electron damping, particularly in the outer plasma layers, at the expense of IC heating. The opposite effect, on the other hand, is to be expected for slower partial waves which would be in resonance with an appreciable number of trapped electrons. For such waves toroidicity tends to depress transit time magnetic 
damping (TTMD) more than it affects electron Landau damping (ELD) and the mixed term (MXT) discussed by Stix [12], thereby altering the balance between the two electron absorption channels, and appreciably reducing the total electron damping. This latter effect, however, is dominant only for waves with relatively large toroidal wavenumbers, which are not much represented in well-tailored toroidal antenna power spectra. Its importance, moreover, could be somewhat overestimated here by the fact that we have completely neglected absorption by trapped electrons. On the whole, therefore, we find that taking into account toroidicity usually leads to predict an increase of direct hf power absorption by the electrons, which in reactorgrade plasmas can be far from negligible, and should be taken into account when estimating the competition between ion and electron heating, and in particular the efficiency of current drive.

\section{The electron hf current in the Ion Cyclotron range of frequencies}

In axisymmetric toroidal geometry it is convenient to represent hf fields and currents as a superposition of toroidal and poloidal Fourier modes

$$
\vec{E}(\psi, \vartheta, \varphi ; t)=\sum_{m, n} \vec{E}^{m n}(\psi) e^{i(m \vartheta+n \varphi-\omega t)}
$$

$(\psi \sim r / a$, with $a$ the minor radius, labels magnetic surfaces; $\vartheta$ and $\varphi$ are a poloidal and the geometric toroidal angle, respectively). In axisymmetric plasmas toroidal modes propagate independently, while poloidal modes are strongly coupled by toroidicity. Solving the linearized Vlasov equation using this spectral representation, the Finite Larmor Radius (FLR) electron current in the Ion Cyclotron and Lower Hybrid (LH) range of frequencies is found to be

$$
\begin{aligned}
& \frac{4 \pi i \omega}{c^{2}} \vec{\jmath}_{e}(\psi, \vartheta, \varphi ; t)=\sum_{m, n} e^{i(m \vartheta+n \varphi-\omega t)} \sum_{\bar{m}}\left\{\frac{\omega^{2}}{c^{2}} \hat{P}_{e}\left(k_{\zeta}^{\bar{m} n}, \psi, \vartheta\right) E_{\zeta}^{m-\bar{m}, n}(\psi) \vec{b}\right. \\
&- \vec{\nabla}_{\perp}^{m n} \times\left[2 \hat{\tau}_{e}\left(k_{\zeta}^{\bar{m} n}, \psi, \vartheta\right)\left(\vec{\nabla}_{\perp}^{m-\bar{m} n} \times \vec{E}_{\perp}^{m-\bar{m}, n}(\psi)\right)\right] \\
&+i\left[\vec{\nabla}_{\perp}^{m n} \times\left[\hat{\xi}\left(k_{\zeta}^{\bar{m} n}, \psi, \vartheta\right) \nabla_{\|}^{m-\bar{m} n} E_{\zeta}^{m-\bar{m}, n}(\psi) \vec{b}\right]\right. \\
&\left.\left.+\nabla_{\|}^{m n}\left[\hat{\xi}_{e}^{\dagger}\left(k_{\zeta}^{\bar{m} n}, \psi, \vartheta\right) \vec{b} \cdot\left(\vec{\nabla}_{\perp}^{m-\bar{m} n} \times \vec{E}_{\perp}^{m-\bar{m}, n}(\psi)\right)\right] \vec{b}\right]\right\}
\end{aligned}
$$

where $m$ and $\bar{m}$ are the poloidal number of the Fourier representation of the current and of the wave fields, respectively. The first line is the contribution of order zero in the electron Larmor radius, whose non-Hermitian part describes Electron Landau Damping (ELD); the second line is the FLR term describing Electron TTMD; and the third is the 'mixed' FLR contribution identified by Stix [12]. The operators $\vec{\nabla}_{\perp}^{m n} \times$ and $\nabla_{\|}^{\bar{m} n}$ are the appropriate components respectively of the curl 
and of the gradient, expressed in the local field-aligned frame, and applied to the $(m, n)$ component of the hf current (outer summation) and to the $(\bar{m}, n)$ component of the wave electric field (inner summation); their explicit form is given in [13]. The coefficients $\hat{P}_{e}, \hat{\tau}_{e}, \hat{\xi}_{e}$, and $\hat{\xi}_{e}^{\dagger}$ have the form

$$
\begin{aligned}
& \hat{P}_{e}\left(k_{\zeta}^{\bar{m}, n}, \psi, \vartheta\right)=-\frac{\omega_{p e}^{2}}{\omega^{2}} \mathcal{I}_{z z}\left(k_{\zeta}^{\bar{m}, n}, \psi, \vartheta\right) \\
& \hat{\tau}_{e}\left(k_{\zeta}^{\bar{m}, n}, \psi, \vartheta\right)+\hat{\tau}_{i}(\psi)=\frac{1}{2} \frac{\omega_{p e}^{2}}{\omega^{2}} \frac{v_{t h e}^{2}}{c^{2}} \mathcal{I}_{y y}\left(k_{\zeta}^{\bar{m}, n}, \psi, \vartheta\right) \\
& \hat{\xi}_{e}\left(k_{\zeta}^{\bar{m}, n}, \psi, \vartheta\right)=-\frac{1}{2} \frac{\omega_{p e}^{2}}{\Omega_{c e} \omega} \frac{v_{t h e}^{2}}{c^{2}} \mathcal{I}_{y z}\left(k_{\zeta}^{\bar{m}, n}, \psi, \vartheta\right) \\
& \hat{\xi}_{e}^{\dagger}\left(k_{\zeta}^{\bar{m}, n}, \psi, \vartheta\right)=-\frac{1}{2} \frac{\omega_{p e}^{2}}{\Omega_{c e} \omega} \frac{v_{t h e}^{2}}{c^{2}} \mathcal{I}_{z y}\left(k_{\zeta}^{\bar{m}, n}, \psi, \vartheta\right)
\end{aligned}
$$

Here $\omega_{p e}$ and $\Omega_{c e}$ are the electron plasma and cyclotron frequencies, $v_{\text {the }}=$ $\left(2 T_{e} / m_{e}\right)^{1 / 2}$ the electron thermal speed, and

$$
k_{\zeta}^{\bar{m}, n}(\psi, \vartheta)=\frac{n+\bar{m} / q(\psi)}{R(\psi, \vartheta)}
$$

the effective parallel wavenumber of the Fourier mode $(\bar{m}, n)$, with $q$ the safety factor and $R$ the major radius. Although not constant, $k_{\zeta}^{\bar{m}, n}$ varies only on the scale of the equilibrium MHD configuration, so that in the IC and LH range of frequencies it can be expected to play in the plasma response to the waves the same role as $k_{\|}$ in the uniform limit. The integrals $\mathcal{I}_{\alpha \beta}$ describe parallel space dispersion in toroidal geometry, and are defined by

$$
\mathcal{I}_{i j}\left(k_{\zeta}^{\bar{m}, n}, \psi, \vartheta\right)=-2 \pi i \omega \int_{0}^{\infty} d \epsilon_{v} \int \frac{B}{v_{\|}} F_{e} \mathrm{~d} \mu_{v} \int_{-\infty}^{t} e^{i\left[(\bar{m}+q n)\left(\vartheta^{\prime}-\vartheta\right)-\omega\left(t^{\prime}-t\right)\right]} \Pi_{i j}^{e} \mathrm{~d} t^{\prime}
$$

where $F_{e}\left(\psi, \epsilon_{v}, \mu_{v}\right)$ is the electron distribution function, $\epsilon_{v}=v^{2} / 2$ and $\mu_{v}=v_{\perp}^{2} / 2 B$ are the particle energy and magnetic moment per unit mass, with $B$ the confining magnetic field, and primes indicate that values are taken along the unperturbed orbits of the guiding centers. The elements $\Pi_{\alpha \beta}^{e}$ in (5) are

$$
\begin{aligned}
& \Pi_{y y}^{e}=\left[\left(-\frac{v_{\text {the }}^{2}}{2 F_{e}} \frac{\partial F_{e}}{\partial \epsilon_{v}}\right)+\frac{1}{B_{0}^{\prime}}\left(1-\frac{k_{\zeta}^{\bar{m} n} v_{\|}^{\prime}}{\omega}\right)\left(-\frac{v_{\text {the }}^{2}}{2 F_{e}} \frac{\partial F_{e}}{\partial \mu_{v}}\right)\right] \frac{w^{2} w^{\prime 2}}{2} \\
& \Pi_{y z}^{e}=2 \frac{\omega}{k_{\zeta}^{\bar{m} n} v_{t h e}}\left(-\frac{v_{\text {the }}^{2}}{2 F_{e}} \frac{\partial F_{e}}{\partial \epsilon_{v}}\right) w^{2} u^{\prime} \\
& \Pi_{z y}^{e}=2 \frac{\omega}{k_{\zeta}^{\bar{m} n} v_{t h e}}\left[\left(-\frac{v_{t h e}^{2}}{2 F_{e}} \frac{\partial F_{e}}{\partial \epsilon_{v}}\right)+\frac{1}{B_{0}^{\prime}}\left(1-\frac{k_{\zeta}^{\bar{m} n} v_{\|}^{\prime}}{\omega}\right)\left(-\frac{v_{t h e}^{2}}{2 F_{e}} \frac{\partial F_{e}}{\partial \mu_{v}}\right)\right] u w^{\prime 2} \\
& \Pi_{z z}^{e}=2\left(-\frac{v_{t h e}^{2}}{2 F_{e}} \frac{\partial F_{e}}{\partial \epsilon_{v}}\right) u u^{\prime}
\end{aligned}
$$

with $u=v_{\|} / v_{\text {the }}, w=v_{\perp} / v_{\text {the }}$, and it is assumed that a 'thermal velocity' $v_{\text {the }}$ can be meaningfully defined even if $F_{e}$ is not a Maxwellian. The terms proportional to 
$\partial F_{e} / \partial \mu_{v}$ vanish at resonance, and, therefore, do not contribute to wave absorption; as a consequence of the large charge to mass ratio of the electrons, moreover, the anisotropy of $F_{e}$ is always very small, i.e. $\partial F_{e} \partial \mu_{v} \approx 0$. Thus eqn (6) can be simplified to

$$
\begin{gathered}
\Pi_{i j}^{e}=\left(-\frac{v_{t h e}^{2}}{2 F_{e}} \frac{\partial F_{e}}{\partial \epsilon_{v}}\right) \Pi_{i j} \\
\Pi_{y y}=\frac{w^{2} w^{\prime 2}}{2} \quad \Pi_{y z}=2 \frac{\omega}{k_{\zeta}^{\bar{m}, n} v_{t h}} w^{2} u^{\prime} \quad \Pi_{z y}=2 \frac{\omega}{k_{\zeta}^{\bar{m}, n} v_{t h}} u w^{\prime 2} \quad \Pi_{z z}=2 u u^{\prime}
\end{gathered}
$$

In the uniform plasma with Maxwellian electrons the integrals (5) can be expressed in terms of the Plasma Dispersion Function (PDF) [14],

$$
Z\left(x_{0}\right)=-\frac{1}{\sqrt{\pi}} \int_{-\infty}^{+\infty} \frac{e^{-u^{2}}}{u-x_{0}} \mathrm{~d} u \quad x_{0}=x_{0}^{\bar{m} n}=\frac{\omega}{k_{\zeta}^{\bar{m} n} v_{\text {the }}}
$$

and its derivatives. We will call the integrals (5) the Toroidal PDFs (TPDFs). We recall that $\Pi_{y y}$ enters the evaluation of TTM damping, $\Pi_{z z}$ the evaluation of ELD, while $\Pi_{x y}$ and $\Pi_{x y}$ enter the so-called mixed (MXD) term which determines the wave polarization [12].

Toroidicity has two consequences on the electron response to the waves. Because of the poloidal variation of $k_{\zeta}^{\bar{m}, n}$ and the modulation of the parallel velocity by the toroidal magnetic field well, Cerenkov-Landau resonances are localized in space rather than in velocity. Because of the periodicity of the configuration, moreover, resonances with harmonics of the bounce or transit frequency $\omega_{B}$ of the parallel motion are possible, which do not exist in the uniform, infinite limit. In the IC range of frequencies the ratio $\omega / \omega_{B}$ is too large for these resonances to contribute directly to the efficiency of wave absorption by the electrons, but their cumulative effect nevertheless influences the electron response to the waves.

\section{Modelling the effects of periodicity on Cerenkov-Landau resonances}

To investigate the effects of the periodic toroidal modulation of the electron parallel velocity on Cherenkov-Landau resonances, a short reminder of the parallel motion of charged particles in axisymmetric toroidal configurations will be useful. At frequencies much higher than the bounce or transit frequency of the electrons in the torus the unperturbed orbits in eqn (5) can be evaluated as if the guiding centers were tied to lines of force of the static magnetic field (this is already implicit in the way the phase of the exponential in (5) has been written). The unperturbed equation of motion is then

$$
\vartheta_{\mathrm{t}}=\sigma_{v} \varpi_{B} \frac{R_{0}}{R}\left(1-\Lambda_{v} \frac{B}{B_{\text {eq }}}\right)^{1 / 2} \quad \text { with } \quad \varpi_{B}=\frac{\sqrt{2 \epsilon_{v}}}{q R_{0}} \ll \omega
$$


where subscript $t$ stands for the time derivative, $R$ the horizontal distance from the vertical axis, $R_{0}$ its value at the magnetic axis, and

$$
\Lambda_{v}=B_{\mathrm{eq}} \frac{\mu_{v}}{\epsilon_{v}}=\frac{v_{\perp \text { eq }}^{2}}{v^{2}}
$$

$\left(0 \leq \Lambda_{v} \leq 1\right)$. Here and in the following the subscript "eq" denotes quantities evaluated at the outer equatorial plane, while quantities evaluated at the magnetic axis will have a subscript "0". In (9), $\sigma_{v}$ is the sign of the parallel velocity on the outer midplane plane $\left(v_{\|}\right.$changes sign along the orbit only in the case of trapped electrons), and $\varpi_{B}$ is the highest angular transit frequency of electrons of energy $m_{e} \epsilon_{v}$, namely those with $\Lambda_{v}=0$ (i.e. $v_{\|}=v, v_{\perp}^{2}=0$ ). The argument of the square root in (9) is always smaller than unity, and vanishes if $\Lambda_{v}=\Lambda_{\mathrm{tp}}=B_{\text {eq }} / B_{\max }$; electrons on the magnetic surface $\psi$ with $\Lambda_{v}>\Lambda_{\text {tp }}$ are toroidally 'trapped' and do not explore the whole magnetic surface.

To second order accuracy in the inverse aspect ratio $\varepsilon_{r}=r / R_{0}$ the intensity of the static magnetic field can be approximated as

$$
B(\psi, \vartheta) \approx B_{0}\left(1-\varepsilon_{r} \cos \vartheta\right)=B_{\text {eq }}\left(1+\frac{\varepsilon_{r}}{1-\varepsilon_{r}}(1-\cos \vartheta)\right) \quad \epsilon_{r}=\frac{a}{R_{0}} \psi
$$

where $a$ is the minor radius, and $\epsilon_{r}$ is the inverse aspect ratio of the magnetic surface $\psi$. Then eqn (9) becomes

$$
\begin{aligned}
& \frac{\vartheta_{\mathrm{t}}}{\omega}=\sigma_{v} \tilde{\eta}_{B}\left(1-\Lambda_{v}\right)^{1 / 2}\left(1-\epsilon_{r} \cos \vartheta\right)\left(1-\frac{1}{2 \kappa_{T}}(1-\cos \vartheta)\right)^{1 / 2} \\
& \text { with } \frac{1}{2 \kappa_{T}}=\frac{\varepsilon_{r}}{1-\varepsilon_{r}} \frac{\Lambda_{v}}{1-\Lambda_{v}}=\frac{\varepsilon_{r}}{1-\varepsilon_{r}} \frac{v_{\perp \text { Le }}^{2}}{v_{\| \text {eq }}^{2}} \text { and } \tilde{\eta}_{B}=\frac{\varpi_{B}}{\omega}
\end{aligned}
$$

$\left(\kappa_{T}=1\right.$ is the transition between trapping and passing). Explicit solutions of this equation which could be used in the orbit integrals of the previous section are not available (cf., however, the discussion on the accuracy at the end of this section), and numerical solutions are manifestly far too cumbersome to be used in TORIC. We can simplify our task, however, by taking advantage of the fact that in the TPDF the orbit integrals are in turn integrated over velocity space. It is, therefore, permissible to use for the orbits approximate expressions which are not uniformly valid in velocity space, provided that the contribution of the velocity domain where they fail is sufficiently small. For this purpose, we must discuss separately wellpassing and deeply trapped particles.

a) Passing electrons. For well passing electrons, $\kappa_{T} \gg 1$, the primed orbits to be used in the GTPDFs (5) can be obtained by solving (12) iteratively:

$$
\vartheta^{\prime}=\vartheta+\omega_{B P}\left(t^{\prime}-t\right)+\epsilon_{B P}\left[\sin \left(\vartheta+\omega_{B P}\left(t^{\prime}-t\right)\right)-\sin \vartheta\right]
$$


with

$$
\omega_{B P}=\frac{v_{\| \mathrm{eq}}}{q R_{0}}\left(1-\frac{1}{4 \kappa_{T}}\right) \quad \epsilon_{B P}=\epsilon_{r}-\frac{1}{4 \kappa_{T}}
$$

Recalling that $k_{\zeta}^{\bar{m} n}$ given in (4) is the parallel component of the local wavevector of the Fourier mode $\vec{E}^{\bar{m}, n}(\psi)$, the exponential in the orbit integrals (5) becomes

$$
\begin{aligned}
& \exp \left\{i\left[(\bar{m}+q n)\left(\vartheta^{\prime}-\vartheta\right)-\omega\left(t^{\prime}-t\right)\right]\right\}= \\
& \quad=\exp \left\{i\left[\frac{(\bar{m}+q n) v_{\| \text {eq }}}{q R_{0}}-\omega\right]\left(t^{\prime}-t\right)+\Xi_{B}^{\bar{m} n}\left(\psi, \Lambda_{v}\right)\left[\sin \left(\vartheta+\frac{v_{\| \text {eq }}}{q R_{0}}\left(t^{\prime}-t\right)\right)-\sin \vartheta\right]\right\} \\
& \quad=\sum_{k, \ell} J_{k}\left(\Xi_{B}^{\bar{m} n}\right) J_{\ell}\left(\Xi_{B}^{\bar{m} n}\right) e^{i(k-\ell) \vartheta} e^{i\left(k_{\zeta}^{\bar{m}+\ell, n} v_{\| \text {eq }}-\omega\right)\left(t^{\prime}-t\right)}
\end{aligned}
$$

with

$$
\Xi_{B}^{\bar{m} n}\left(\psi ; \Lambda_{v}\right)=\epsilon_{B P}(\bar{m}+q n) \simeq \epsilon_{r}-\frac{1}{2} \frac{\epsilon_{r}}{1-\epsilon_{r}} \frac{\Lambda_{v}}{1-\Lambda_{v}}(\bar{m}+q n)
$$

Note the close similarity of this equation to the expressions obtained by expanding the gyromotion contribution to the phase in harmonics of the cyclotron frequency (whose role is taken here by the bounce frequency). There is one important complication, however: the 'gyration angle' is in velocity space, and in the GTPDF is integrated over; the 'bounce angle', by contrast, is in space, and since the GTPDF is needed at all poloidal points separately, the double Bessel series in eqn (15) cannot be diagonalized.

The integral over $t^{\prime}$ can nevertheless be performed, since to the present accuracy we can also omit the primes in the factors $\Pi_{i j}$. The contribution of passing electrons to the GTPDF can then be estimated as

$$
\begin{aligned}
\mathcal{I}_{i j}^{P}\left(k_{\zeta}^{\bar{m}, n}, \psi, \vartheta\right)=2 \pi \int_{0}^{\infty} & \mathrm{d} \epsilon_{v} \int_{\left(\kappa_{T}>1\right)} \frac{B_{0}}{v_{\|}} F_{e} \Pi_{i j} \mathrm{~d} \mu_{v} \\
& \sum_{k, \ell} J_{k}\left(\Xi_{B}^{\bar{m} n}\right) J_{\ell}\left(\Xi_{B}^{\bar{m} n}\right) e^{i(k-\ell) \vartheta} \frac{\omega}{\omega-k_{\zeta}^{\bar{m}+\ell, n} v_{\|}}
\end{aligned}
$$

with the $\mu_{v}$-integral extending over all passing particles $\left(\kappa_{T}>1\right)$. The summation over $\ell$ with resonant denominators can be regarded as a broadening of the Cherenkov resonance due to the cumulative effect of resonances with the periodic parallel motion of the guiding centers.

b) Trapped electrons. To lowest order in the inverse aspect ratio the condition for a particle to be toroidally trapped is

$$
\Lambda_{v}>\Lambda_{\mathrm{tp}} \simeq \frac{1-\varepsilon_{r}}{1+\varepsilon_{r}}
$$

Roughly speaking, a particle is deeply trapped if it satisfies $1-\Lambda_{v}<\epsilon_{v}$ by a sufficient margin; the turning point is then

$$
\vartheta_{\mathrm{tp}} \simeq 2 \sqrt{\kappa_{T}}=\left(\frac{2\left(1-\varepsilon_{r}\right)}{\varepsilon_{r}}\left(1-\Lambda_{v}\right)\right)^{1 / 2} \quad 1-\Lambda_{v} \ll \varepsilon_{r}
$$


In the domain explored by a deeply trapped particle, moreover,

$$
\frac{B}{B_{\mathrm{eq}}} \simeq 1+\varepsilon_{r}(1-\cos \vartheta) \simeq 1+\varepsilon_{r} \frac{\vartheta^{2}}{2}
$$

Under these conditions eqn (9) becomes the equation of a linear oscillator around $\vartheta=0$,

$$
\frac{\vartheta_{t}^{2}}{\vartheta_{\mathrm{tp}}^{2}} \simeq \omega_{B T}^{2}\left(1-\frac{\vartheta^{2}}{\vartheta_{\mathrm{tp}}^{2}}\right) \quad \omega_{B T}=\omega_{B}\left(\varepsilon_{r} / 2\right)^{1 / 2} \simeq\left(\frac{1-\varepsilon_{r}}{2 \varepsilon_{r}}\right)^{1 / 2} \frac{v_{\| \mathrm{eq}}}{q R_{0}}
$$

Indeed, deriving both members with respect to $t$ we obtain the linear equation

$$
\vartheta_{t t}=-\omega_{B T}^{2} \vartheta
$$

whose solution compatible with $(21)$ is

$$
\vartheta=\vartheta_{\mathrm{tp}} \sin \omega_{B T}\left(t-t_{\mathrm{eq}}\right)
$$

where $t_{0}$ is the time of the last transit through the outer equatorial plane.

The presence of the 'privileged' time $t_{\mathrm{eq}}$ in eqn (23) makes it rather cumbersome to write this solution in a form appropriate for the phase of the exponential in the orbit integrals. To eliminate $t_{\text {eq }}$ we can write

$$
\begin{aligned}
\vartheta^{\prime}-\vartheta & =\vartheta_{\mathrm{tp}}\left[\sin \omega_{B T}\left(t^{\prime}-t_{\mathrm{eq}}\right)-\sin \omega_{B T}\left(t-t_{\mathrm{eq}}\right)\right] \\
& =\left(\vartheta_{\mathrm{tp}}^{2}-\vartheta^{2}\right)^{1 / 2} \sin \left(\omega_{B T}\left(t^{\prime}-t\right)\right)+\vartheta\left(\cos \left(\omega_{B T}\left(t^{\prime}-t\right)\right)-1\right)
\end{aligned}
$$

Accordingly, the exponential in the trapped electrons contribution to the orbit integral can be expanded as the product of two series in harmonics of $\omega_{B T}$

$$
\begin{gathered}
\exp \left\{i\left[(\bar{m}+q n)\left(\vartheta^{\prime}-\vartheta\right)-\omega\left(t^{\prime}-t\right)\right]\right\}=\sum_{r, s} J_{r}\left(\Xi_{\mathrm{tp}}^{\bar{m} n}\right) \mathcal{C}_{s}\left(\Xi_{\vartheta}^{\bar{m} n}\right) e^{i(r+s) \omega_{B T}\left(t^{\prime}-t\right)} \\
\mathcal{C}_{2 s}=(-1)^{s} J_{2 s}\left(\Xi_{\vartheta}^{\bar{m} n}\right) \cos \Xi_{\vartheta}^{\bar{m} n} \\
\mathcal{C}_{2 s+1}=(-1)^{s+1} J_{2 s+1}\left(\Xi_{\vartheta}^{\bar{m} n}\right) \sin \Xi_{\vartheta}^{\bar{m} n}
\end{gathered}
$$

with

$$
\Xi_{\mathrm{tp}}^{\bar{m} n}=(\bar{m}+q n)\left(\vartheta_{\mathrm{tp}}^{2}-\vartheta^{2}\right)^{1 / 2} \quad \Xi_{\vartheta}^{\bar{m} n}=(\bar{m}+q n) \vartheta
$$

Recalling the definition (21) of $\omega_{B T}$, the contribution of trapped electrons to the GTPDF can then be approximated as

$$
\begin{gathered}
\mathcal{I}_{i j}^{T}\left(k_{\zeta}^{\bar{m}, n}, \psi, \vartheta\right)=2 \pi \int_{0}^{\infty} \mathrm{d} \epsilon_{v} \int_{\left(\kappa_{T}>1\right)} \frac{B_{0}}{v_{\|}} F_{e} \Pi_{i j} \mathrm{~d} \mu_{v} \sum_{r, s} J_{r}\left(\Xi_{\mathrm{tp}}^{\bar{m} n}\right) \mathcal{C}_{s}\left(\Xi_{\vartheta}^{\bar{m} n}\right) \frac{\omega}{\omega-(r+s) k_{T} v_{\perp \text { eq }}} \\
k_{T}=\sqrt{\frac{\epsilon_{r}}{2\left(1-\epsilon_{r}\right)}} \frac{1}{q R_{0}}
\end{gathered}
$$


with the $\mu_{v}$-integral extending over all trapping particles $\left(\kappa_{T}<1\right)$.

The space and velocity dependence of the integrand of eqn (27) makes its numerical evaluation prohibitively lengthy; from this equation we can nevertheless derive a useful conclusion. The fact that trapped electrons are very slow along magnetic field lines is reflected in the factor $\left(\varepsilon_{r} / 2\right)^{1 / 2} \ll 1$ affecting $k_{T}$ in the resonant denominators. Thus trapped electrons can resonate only with waves with a very slow parallel phase velocity. On the other hand, the inequality $\left(q R_{0}\right)^{-1} \ll\left|k_{\zeta}^{\bar{m}, n}\right|$ is always satisfied by a large margin in practice. This means that if the antenna spectrum is tailored to resonate with electrons with parallel velocities of the order or a few times larger than the thermal speed, as is normally the case, very few trapped electrons will experience a Cerenkov resonance. We conclude that FW wave absorption by trapped electrons will usually play a very marginal role.

c) Implementation in the TORIC code. We take advantage of the last observation to build a model sufficiently simple to be easily implemented in a full-wave solver of Maxwell's equations in toroidal geometry, yet reasonably accurate. For this purpose we introduce the following simplifications:

(i) We will take into account the effects of the periodic modulation of the electron parallel velocity only in the imaginary part of the GPDFs, responsible for wave absorption;

(ii) We will use the expression (17) for the contribution of passing electrons, and neglect completely the contribution of trapped electrons.

The last assumption is certainly questionable: the contribution of trapped electrons to the absorption, although small, is not zero. It can nevertheless be justified by stressing again that in the domain of velocity space in which eqn (17) does not apply very few resonant electrons should normally be present.

With the above approximations the velocity space integrals in eqn (17) can be performed using cylindrical velocity coordinates, obtaining

$$
\mathcal{I}_{i j}\left(k_{\zeta}^{\bar{m}, n} ; \psi, \vartheta\right)=-x_{0}^{\bar{m}, n}\left[\operatorname{Re}\left(\mathcal{Z}_{i j}\left(x_{0}^{\bar{m} n}\right)\right)+i \Gamma_{i j}\left(x_{0}^{\bar{m}, n}, \psi, \vartheta\right)\right]
$$

with real part

$$
\operatorname{Re}\left(\mathcal{Z}_{i j}\left(x_{0}^{\bar{m} n}\right)\right)=\frac{1}{\sqrt{\pi}} \mathrm{P} \int_{-\infty}^{\infty} \frac{e^{-u^{2}} \mathrm{~d} u}{u-x_{0}^{\bar{m} n}} \int_{0}^{\infty} e^{-w^{2}} \Pi_{i j}(u, w) w \mathrm{~d} w
$$

(the singular integral to be evaluated as in section 2), and imaginary part

$$
\Gamma_{i j}\left(x_{0}^{\bar{m}, n}, \psi, \vartheta\right)=\sqrt{\pi} \sum_{k, \ell} \mathcal{F}_{i j}^{k, \ell}\left(x_{0}^{\bar{m}+\ell, n} ; \psi, \vartheta\right) \cos (k-\ell) \vartheta
$$


with

$$
\begin{array}{r}
\mathcal{F}_{i j}^{k, \ell}\left(x_{0}^{\bar{m}+\ell, n} ; \psi, \vartheta\right)=e^{-\left(x_{0}^{\bar{m}+\ell, n}\right)^{2}} \int_{\Lambda_{v}<\Lambda_{\mathrm{tp}}} e^{-w^{2}} \Pi_{i j}\left(x_{0}^{\bar{m}+\ell, n}, w\right) J_{k}\left(\Xi_{B}^{\bar{m}+\ell, n}\right) J_{\ell}\left(\Xi_{B}^{\bar{m}+\ell, n}\right) w \mathrm{~d} w \\
\Xi_{B}^{\bar{m}+\ell, n}=\varepsilon_{r}-\frac{1}{2} \frac{w^{2}}{\left(x_{0}^{\bar{m}+\ell, n}\right)^{2}} \frac{\varepsilon_{r}}{1-\varepsilon_{r}}(\bar{m}+q n)
\end{array}
$$

(the function $\mathcal{F}$ depends on $\vartheta$ through the value of $x_{0}^{\bar{m}+\ell, n}$ ). In the limit $\epsilon_{r} \rightarrow 0$ one recovers the uniform-plasma result, as should be expected. Two further comments about these results are worth being mentioned:

1) According to (30), $\Gamma_{i j}$ is stationary at the equatorial plane: it is maximum (in absolute value) at $\vartheta=0$, and minimum at $\vartheta=\pi$. It follows that the toroidal effects on damping by the electrons are strongest just in front of the antenna, a fact that under appropriate conditions can appreciably amplify their influence on the global power balance.

2) The integral over perpendicular velocity in (31) is performed excluding the domain of trapped electrons. Due to the different weight of the quantities $\Pi_{i j}$ with $w$, this reduces the (absolute) value of $\Gamma_{y y}$ (TTMD) more than that $\Gamma_{z z}$ (ELD) and of $\Gamma_{x y}$ and $\Gamma_{y x}(\mathrm{MXD})$. The effect decreases with increasing parallel phase velocity of the wave, because the number of trapped electrons in the excluded domain decreases exponentially.

d) Some considerations about the accuracy. As already noted, no analytical solution of eqn (9) is available to which our approximate solutions could be compared. However, an approximate but completely explicit description of the parallel motion of charged particles in the tokamak magnetic field (11) in terms of elliptic integrals becomes possible [15] if the factor $R_{0} / R$ outside the square root in eqn (9) is omitted. In this subsection, therefore, we explore the accuracy of our approximate solutions by comparing them with the solutions obtained in the literature in terms of elliptic functions.

Before proceeding, it is worth stressing that the just mentioned factor $R_{0} / R$ in eqn (9) is essential for our problem, and cannot be omitted. In the first place, the inaccuracy introduced is already of first order in the inverse aspect ratio. More important, however, is the fact that if $\vartheta_{\mathrm{t}}$ is written without this factor one finds that the second derivative $(m+q n) \vartheta_{\text {tt }}$ of the wave phase seen by an electron along its orbit would be proportional to $\Lambda_{v}$, implying $\vartheta_{\text {tt }} \equiv 0$ for $v_{\perp}=0$, i.e. to lowest order in the inverse aspect ratio one would predict no localization of the Cerenkov resonance of a strictly passing electron. This is in obvious contradiction with the resonance condition $k_{\|} v_{\|}=\omega$, which states that for such an electron, which has a constant $v_{\|}=v$, localization and duration of the resonance are determined to lowest order by the $\sim 1 / R$ variation of $k_{\|}$along the orbit. The outer factor in eqn (9) corresponds precisely to the $R^{-1}$ variation of $k_{\|}$: in other words, writing the resonance condition 

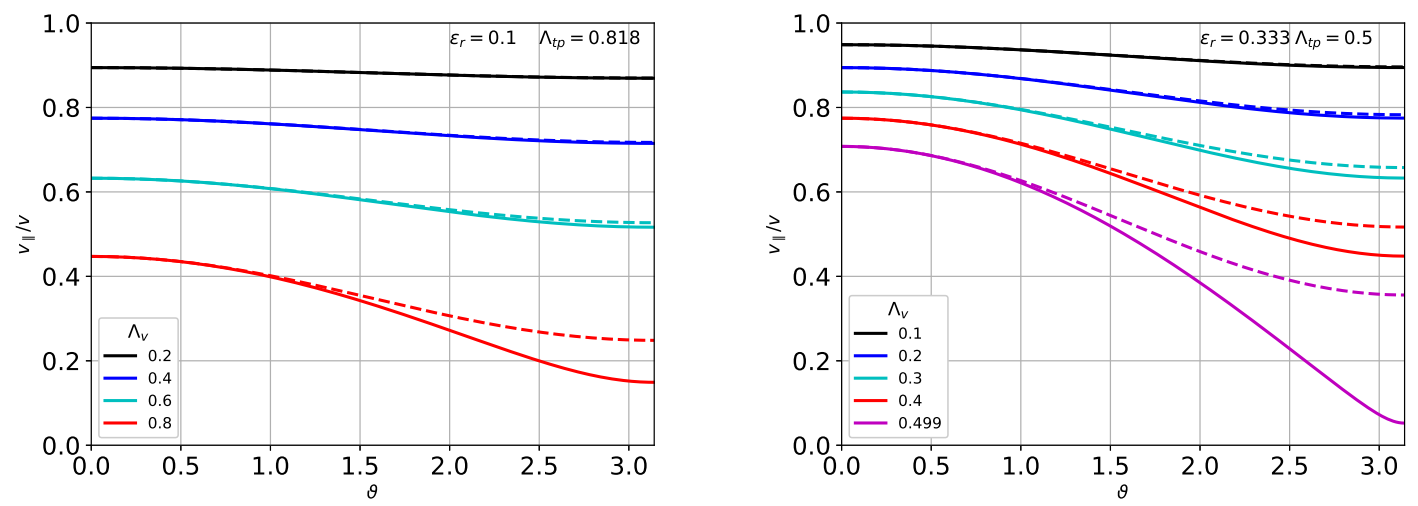

Figure 1. Parallel velocity of passing electrons in the tokamak magnetic field as function of the poloidal angle $\vartheta$. The dashed lines correspond to the approximation (28).

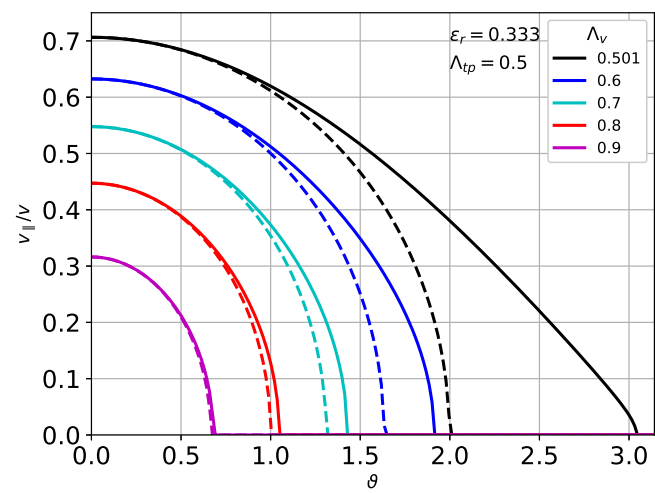

Figure 2. Parallel velocity of trapped particles in the tokamak magnetic field as function of the poloidal angle $\vartheta$. The dashed lines correspond to the approximation (2).

in terms of the time history of the poloidal angle displaces the $R^{-1}$ dependence of $k_{\|}$ into the $R^{-1}$ dependence of $\vartheta_{\mathrm{t}}$. Omitting this factor, therefore, leads to a completely wrong estimate of the duration of Cerenkov resonances, and, therefore, of the effects of toroidicity on electron Landau resonances.

For our purposes here, we have circumvented this difficulty by comparing, instead of the solutions for the poloidal angle, the solutions for the parallel velocity, assuming that in the analytic case the proportionality factor between $\vartheta_{\mathrm{t}}$ and $v_{\|}$can be regarded as independent from the poloidal angle, as implicitly assumed in [15]. This comparison is presented in fig. 1 for passing, and in fig. 2 for trapped electrons. As can be deduced from these figures, our approximation are actually acceptable in a wider range of $\Lambda_{v}$ than stipulated above, and fail only in the vicinity of the passing-trapping boundary. The same conclusion can be derived from fig. 3, where 


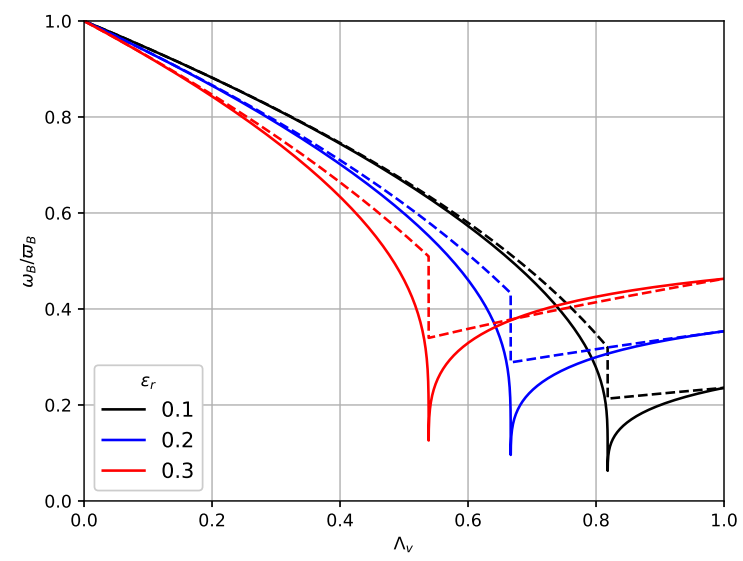

Figure 3. The ratio $\omega_{B} / \varpi_{B}$ as function of $\Lambda_{v}$ for three values of the inverse aspect ratio of the magnetic surface. The dashed curves are the approximation (14) and (21).

the approximations (14) and (21) for the bouncing frequency are compared to the 'exact' expressions in terms of elliptic functions.

\section{Examples}

\subsection{Minority heating in medium-size tokamaks.}

In minority heating scenarios in medium-size devices IC damping almost always largely dominates over damping by the electrons, so that the effects of toroidicity on the unperturbed electron motion do not significantly affect the global power balance. As an example, we have chosen a typical minority heating scenario in a plasma of the dimensions of the ASDEX Upgrade device (Hydrogen minority in Deuterium, central density $6.410^{19} \mathrm{~m}^{-3}$, central temperatures $T_{e}=4.25 \mathrm{keV}, T_{i}=2.28 \mathrm{keV}$, magnetic field on axis $B_{0}=2.4$ Tesla, applied frequency $36.5 \mathrm{MHz}$, representative toroidal mode $n_{\varphi}=12$; the selfconsistent quasilinear ion distributions and power absorption profiles have been calculated assuming a total hf power of 1.6 MW). Taking into account the effects considered in this note increases the fraction of power predicted to be absorbed by the electrons in a Maxwellian plasma from $8 \%$ to $13 \%$, while absorption by the minority is predicted to decrease from $66.20 \%$ to $61.63 \%$, and harmonic absorption by Deuterium from $27.47 \%$ to $25.40 \%$. While nearly doubling of direct absorption of the waves by the electrons might not be regarded as a minor effect, it is well below what can be put into evidence experimentally, in particular because collisional redistribution of the absorbed power complicates the issue.

It is nevertheless interesting to illustrate the role of geometry and of the relative strength of absorption by the different species by varying the assumed minority 


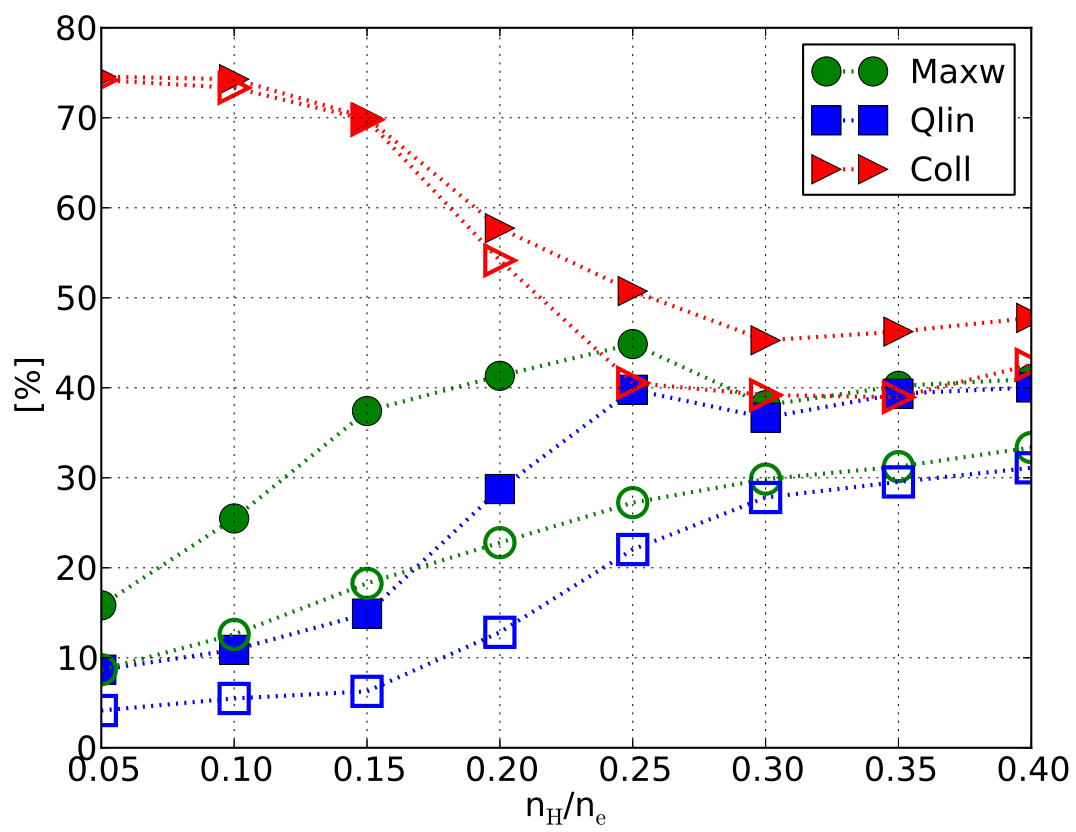

Figure 4. Influence of toroidicity on electron absorption and heating in a mediumsize plasma as function of the minority concentration. Green bullets: Maxwellian plasma; blue squares: selfconsistent quasilinear ion distribution functions; red triangles: same after collisional redistribution. Open symbols: without toroidicity effects on electron absorption; Full symbols: with toroidicity effects on electron absorption.

concentration in this plasma. The results are summarized in fig. 4. As the Hydrogen concentration increases, screening of $E_{+}$at the IC resonance increases, decreasing IC absorption per transit; multiple transits enhances the impact of toroidicity effects on direct electron absorption. At the same time the energy in the suprathermal Hydrogen population decreases, so that less power is collisionally transferred to the electrons. The effect of toroidicity on direct electron absorption are best visible around and just above the transition from the minority to the mode-conversion regime, which is situated in this case around $15 \%$ minority concentration. In this region, on the other hand, for the reason just mentioned a reduction can be seen in the power gained by the electrons after collisional redistribution.

The effects on the direct absorption profiles are illustrated in fig. 5. They are barely visible in the minority regime, but beyond the transition mode conversion the peak of power deposition in the minority is appreciably lowered, explaining the reduced collisional transfer to the electrons.

It is also interesting to investigate how the impact of toroidicity on direct electron absorption depends on the toroidal wavenumber $n_{\varphi}$, i.e. on the average parallel phase velocity of the wave (because the field of each toroidal mode is a superposition of many coupled poloidal Fourier components, one value of $n_{\varphi}$ corresponds to 

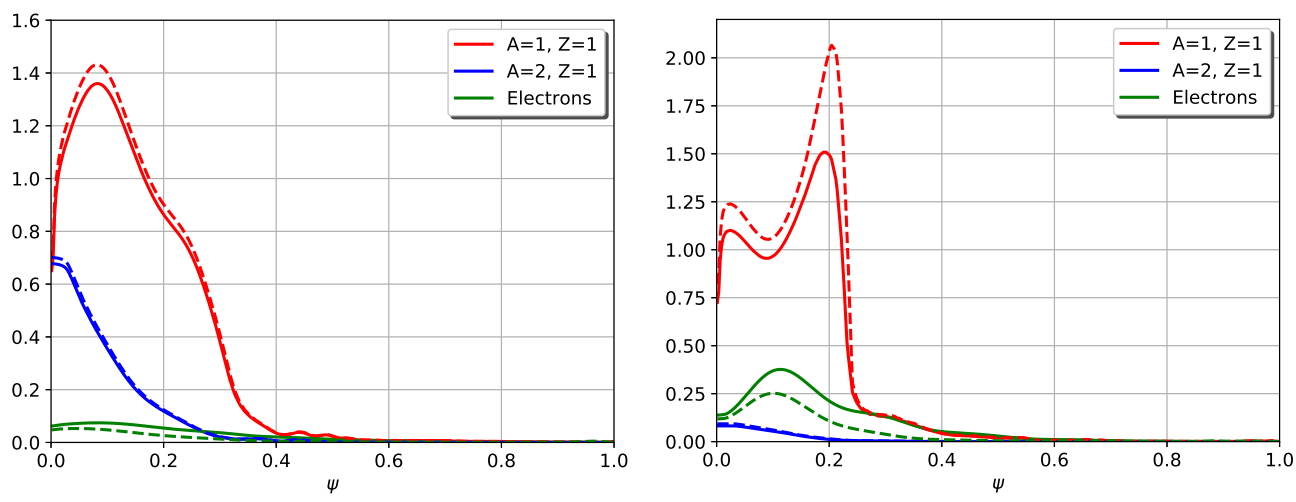

Figure 5. Power absorption profiles in a medium-size plasma, selfconsistent ion distribution functions. Left: 5\% Hydrogen, right: 20\% Hydrogen. Full curves with toroidal effects, dashed curves without toroidal effects.

a spectrum of $k_{\|}$values, which shrinks to $n_{\varphi} / R_{0}$ on the magnetic axis; in our simulations the range of poloidal modes used spanned the range $-15 \leq m \leq+15$ ). For this purpose we have chosen the Hydrogen concentration of $13 \%$, roughly at the transition between minority and mode conversion. In present-day devices the toroidal spectrum of antennas excited in the antisymmetric configuration for ion heating typically spans the range $-30 \lesssim n_{\varphi} \lesssim+30$, with a peak around $n_{\varphi} \sim 12$ to 15. In fig. 6 we present the results for a few selected toroidal modes (only the Maxwellian plasma case has been considered: to iterate to obtain the selfconsistent quasilinear solution makes sense only for a representative mode near the peak of the spectrum, or for the entire spectrum). The increase of direct electron absorption due to toroidicity is particularly spectacular for $n_{\varphi}=6$ : this is due to multi-transit, because this mode is much more weakly damped than modes close to the main peak of the spectrum. Again, this is not something that could easily be seen experimentally, because the weight of this mode in the typical antenna toroidal power spectrum is very small.

We can also note that for values of $n_{\varphi}$ in the upper part of the spectrum (18 and 24) direct electron absorption is depressed in the central region. This is because its enhancement in the periphery is sufficient to appreciably decrease the power reaching the core of the plasma (these modes are damped essentially in a single pass). Once more, this effect would not show up in a simulation covering the entire toroidal spectrum, because the weight of these mode is relatively small.

\subsection{A minority heating scenario in ITER, non-activated phase.}

In fusion-grade plasmas, such as ITER, electron damping is a more serious competitor, because of the higher temperature, and of the thicker plasma layer the waves must cross before reaching IC resonances. 

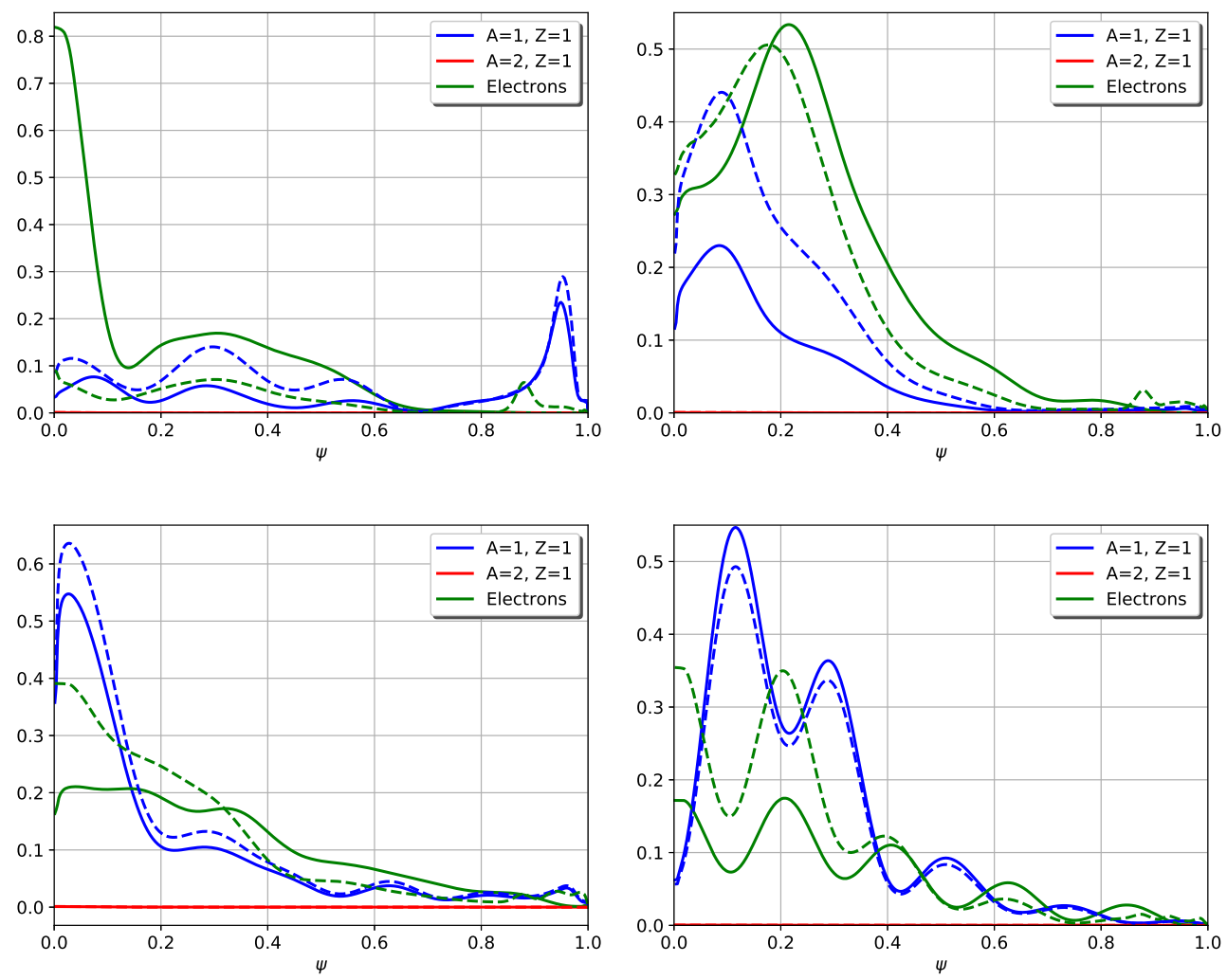

Figure 6. Hydrogen concentration 13\%: power deposition profiles for selected toroidal modes, selfconsistent ion distribution functions. From above left to below right: $n_{\varphi}=6,12,18$, and 24 . Full curves with toroidal effects, dashed curves without toroidal effects.

As an example, we have considered a minority heating scenario in a ${ }^{4} \mathrm{He}$ plasma with a Hydrogen minority (5\% in density, with magnetic field 2.65 Tesla on axis; at $40 \mathrm{MHz}$ the IC resonance are very close to the axis. The central density was assumed $4.910^{19} \mathrm{~m}^{-3}$, with central temperatures $T_{e}=16.7 \mathrm{keV}, T_{i}=15.2 \mathrm{keV}$. With the antenna of the dimensions proposed in [16] and 4 straps, the radiated toroidal power spectrum has two well-defined peaks around $n_{\varphi}= \pm 60$; we have therefore performed the consistency loop between TORIC and SSFPQL for the representative mode $n_{\varphi}=60$ (average $k_{\|} \sim 9.4 \mathrm{~m}^{-1} \ddagger$ ) assuming a total power of $20 \mathrm{MW}$. The predicted power deposition profiles in the Maxwellian plasma and with the selfconsistent quasilinear ion distribution functions are shown in fig. 7.

A new effect can be observed in this case. In the relatively cold plasma near the boundary the situation is similar to that in present day devices, and toroidal

‡ TORIC predicts a launched power spectrum shifted to slightly higher toroidal wavenumbers than those in [16], and in the case of CD configuration a different weight between the positive and negative part of the spectrum; this might depend on the supposed distance of the antenna from the separatrix and the assumed density profile in the plasma periphery. We have checked that from the point of view of the effects discussed here the difference is not significant. 

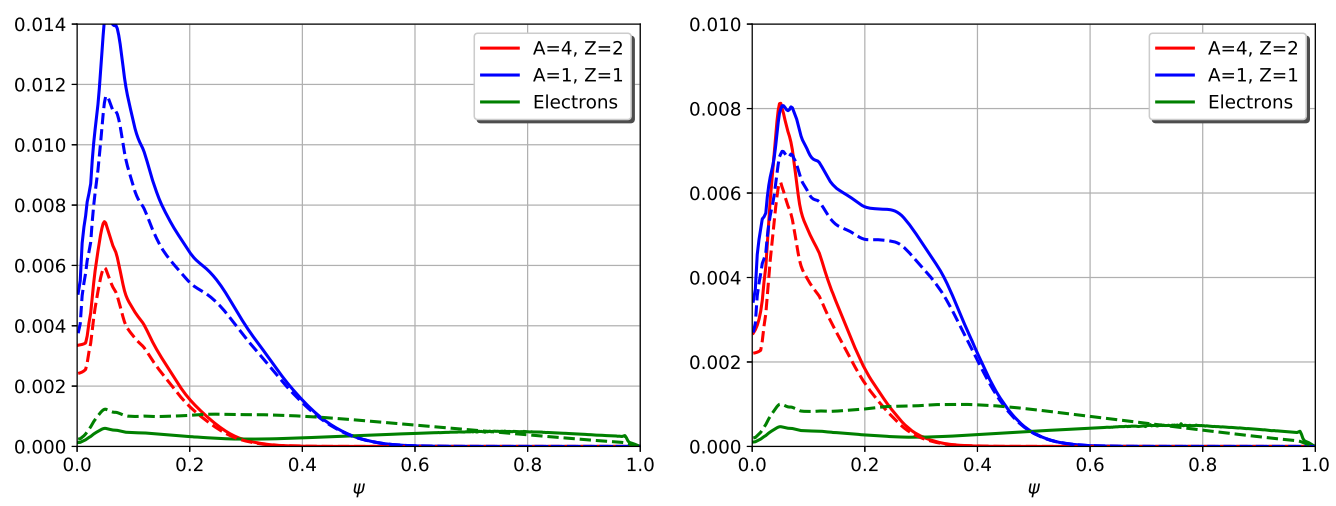

Figure 7. Minority Heating in ITER, $5 \% \mathrm{H}$ in ${ }^{4} \mathrm{He}, n_{\varphi}=60$. Left Maxwellian plasma, right Selfconsistent ion distribution functions. Full curves with toroidal effects, dashed curves without toroidal effects.

effects enhance electron damping. As the temperature increases away from the edge, however, the ratio of the average phase velocity of the waves to the local electron thermal speed decreases (it is around two on axis) so that the fraction of resonant electrons which are toroidally trapped increases, and absorption by the electron is depressed. This more than compensates the reduction in Poynting flux at the transition through the outer layers, so that IC heating in the core is even slightly enhanced. This enhancement is actually not really significant, since it is probably of the same order as the inaccuracy in electron heating due to the complete neglect of absorption by trapped electrons. The behaviour of the electron deposition profile when toroidicity effects are taken into account, on the other hand, is robust, and is not expected to change much if a better model for trapped electrons will become available.

\subsection{A current drive scenario in ITER, non-activated phase.}

In ITER frequency windows for hf current drive in the IC range (ICCD) are difficult to be found $[17,18]$. In the initial non-activated phase, ICCD might be marginally possible at the upper end of the planned frequency range. We have therefore considered the same ${ }^{4}$ He plasma with a residual Hydrogen minority $\sim 3 \%$ in density (well below the optimum for IC minority absorption) at a frequency of $53 \mathrm{MHz}$ : the IC resonances are then close to the inner boundary, so that absorption by the electrons is maximized. In this example we have considered only a Maxwellian plasma, because the density of power deposited in the ions is so low to make quasilinear effects essentially negligible. Although this scenario is unlikely to be really adopted, it is well suited to illustrate the effects discussed in this note.

With a 4 strap antenna excited in the CD configuration, the dominant lobe in the toroidal power spectrum is peaked around $n_{\varphi}=30$, with a secondary lobe peaked 


\begin{tabular}{|c|c|c|c|c|c|c|}
\hline$n_{\varphi}$ & \multicolumn{2}{|c|}{ electrons } & \multicolumn{2}{c|}{ Hydrogen } & \multicolumn{2}{c|}{$\mathrm{He}^{4}$} \\
& w/o & with & w/o & with & w/o & with \\
\hline+30 & 74.43 & 94.39 & 22.65 & 4.96 & 2.91 & 0.65 \\
\hline-90 & 88.91 & 68.04 & 10.69 & 30.69 & 0.40 & 1.27 \\
\hline
\end{tabular}

Table 1. Power repartition without (w/o) and with toroidal trapping with Maxwellian distribution functions.
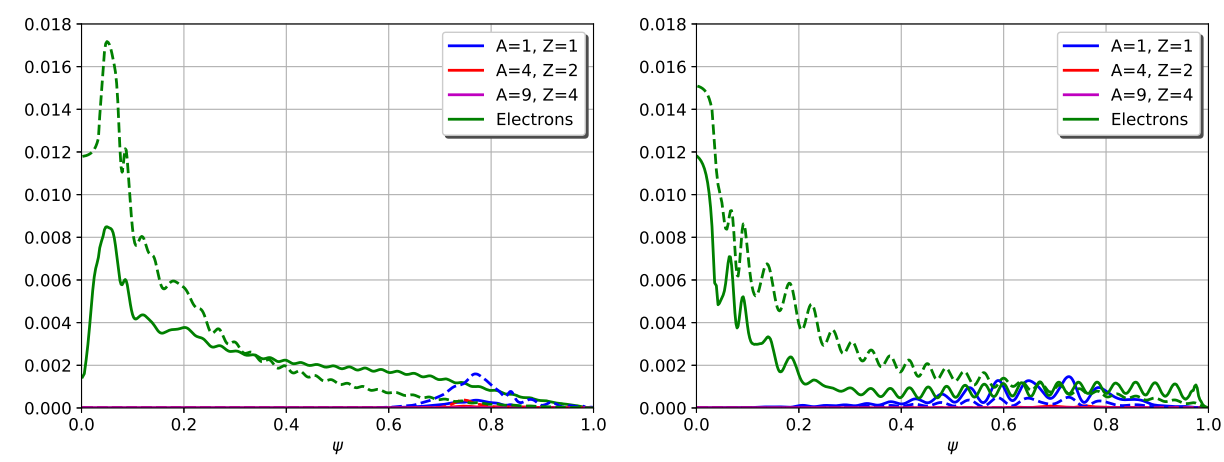

Figure 8. ITER He4 plasma with $3 \%$ Hydrogen, 2.655 Tesla on axis, $53 \mathrm{MHz}$, left $n_{\varphi}=30$, right $n_{\varphi}=-90$. Power deposition profiles in a Maxwellian plasma, normalized to $1 \mathrm{MW}$ total. Dashed lines without, full lines with toroidal effects on the electrons.

around $n_{\varphi}=-90$; depending on the detailed geometry of the antenna, the power launched in the negative direction ranges between one third and half of the power in the positive direction.

As can be seen from table 1, when toroidicity effects on the electrons are taken into account (columns labeled with TE) TORIC predicts a substantial increase of electron absorption of the wave propagating in the positive toroidal direction, and a comparable decrease in electron absorption of the wave traveling in the negative direction. At first sight this appears favorable to current drive. A look to the deposition profiles (fig. 8), however, shows that the increased absorption of the mode $n_{\varphi}=+30$ occurs mainly in the outer half of the plasma: indeed, at this high electron temperature, the increased damping in the plasma periphery is large enough to severely reduce the amount of power reaching the plasma core. Any increase of the driven current, therefore, will be accompanied by a marked displacement of the CD profile towards the outside. The importance of these modifications indicates that to plan and interpret IC and CD experiments in large and hot plasmas attention should be given also to the effects of toroidicity on direct absorption by the electrons. 


\section{Conclusions}

We have developed a model to take into account the effects of the poloidal modulation of the parallel velocity of charged particles in the tokamak magnetic field on the absorption of the Fast Wave by the electrons in the ion cyclotron range of frequency. To make the model numerically tractable it has been necessary to make some rather drastic approximations, which, however, appear acceptable under most situations. The resulting change in the strength of damping by the electrons, although locally moderate, can be amplified by the fact that it is largest in the plasma periphery just in front of the antenna, and because the waves often have to traverse a large volume of plasma were they are subject only to electron damping before reaching IC resonances where they can heat the ions. Thus the effect is usually (although not always) negligible in present-day medium size tokamaks, but its importance is bound to increase with increasing plasma size and electron temperature. In ITER and larger future tokamaks it will be important to take into account these effects when estimating the competition between electron and ion heating, or the efficiency of current drive.

[1] KAladZe, T., Pyatak, A., and Stepanov, K., Sov. J. Plasma Phys. 8 (1982) 467.

[2] GRISHANOV, N. and NEKRASOV, F., Sov. J. Plasma Phys. (1987) 65.

[3] NEKRASOV, F., Sov. J. Plasma Phys. 16 (1990) 35.

[4] ELFIMOV, A. and PURI, S., Nucl. Fusion 30 (1990) 1215.

[5] ELFIMOV, A. and NEKRASOV, F., Sov. J. Plasma Phys. 18 (1992) 526.

[6] GRISHanOV, N., DE AZEVEdO, C., and DE ASSIS, A., Phys. of Plasmas 4 (1997) 1055.

[7] NeKrasov, F., Elfimov, A., DE AZEvedo, C., and DE ASSiS, A., Phys. Letters A 251 (1999) 44.

[8] Grishanov, N., LOUlA, A., DE AZEvedo, C., and NetO, J. P., Plasma Phys. Contr. Fusion 45 (2003) 1791.

[9] GRISHANOV, N. and AZARENKOV, N., Plasmas Phys. Review 39 (2013) 1070.

[10] BRAMBILlA, M. and BILATO, R., Nuclear Fusion 49 (2009) 085004.

[11] BRAMBILla, M., Physics Letters A 188 (1994) 376 .

[12] STIX, T., Nuclear Fusion 15 (1975) 737.

[13] BRAmBilla, M., Plasma Physics and Controlled Fusion 41 (1999) 1.

[14] FRIED, B. D. and CONTE, S. D., The plasma dispersion function, N.Y. : Academic Press, 1961.

[15] BRIZARD, A. J., Physics of Plasmas 18 (2011) 022508.

[16] MESSiAEn, A. and WEYNANTS, R., Plasma Physics and Controlled Fusion 53 (2011) 085020.

[17] HAnnan, A., Hellsten, T., and JOHnSOn, T., Nuclear Fusion 53 (2013) 043005.

[18] BRAmBilla, M. and BILATO, R., Nuclear Fusion 55 (2015) 023016. 